\title{
Tareas que desarrollan el sentido matemático en la formación inicial de profesores
}

\section{Tasks for developing the mathematical sense in the initial training of teachers}

\author{
Juan Francisco Ruiz-Hidalgo ${ }^{1}$ \\ Pablo Flores Martínez ${ }^{2}$ \\ Rafael Ramírez-Uclés ${ }^{3}$ \\ José Antonio Fernández-Plaza ${ }^{4}$
}

\begin{abstract}
Resumen: Desde hace tiempo, diversos organismos, como el National Council of Teachers of Mathematics, promueven una enseñanza de las matemáticas con sentido. Desde nuestra perspectiva de formadores de profesores de matemáticas y basándonos en investigaciones de reconocido prestigio, mostramos en este trabajo un modelo de formación de profesores en el que desarrollamos el sentido matemático a través de tareas matemáticas escolares y de la identificación de objetivos de aprendizaje para dichas tareas. En este modelo, repasamos las capacidades matemáticas necesarias para enseñar matemáticas con sentido, relacionándolas con distintos sentidos matemáticos -numérico, espacial, de la medida y estocástico- y ejemplificamos con tareas de formación que permiten su desarrollo en futuros maestros de educación primaria. Asimismo, estas tareas desarrollan las capacidades que los futuros maestros podrán utilizar en su labor docente, pues se trata de un modelo que va más allá de la memorización de los componentes de los sentidos matemáticos, pretendiendo lograr un conocimiento funcional de las matemáticas.
\end{abstract}

Fecha de recepción: 8 de junio de 2018. Fecha de aceptación: 6 de febrero de 2019 .

${ }_{1}^{1}$ Universidad de Granada, España, jfruiz@ugr.es, orcid.org/0000-0002-4805-6922

2 Universidad de Granada, España, pflores@ugr.es, orcid.org/0000-0002-3292-6639

3 Universidad de Granada, España, rramirez@ugr.es, orcid.org/0000-0002-8462-5897

${ }^{4}$ Universidad de Granada, España, joseanfplaza@ugr.es, orcid.org/0000-0001-6570-0866 
Palabras clave: matemáticas para maestros, sentido matemático, tareas matemáticas, capacidades matemáticas, objetivos de aprendizaje.

\begin{abstract}
For a long time, various agencies, such as the National Council of Teachers of Mathematics, have advocated promoting a meaningful teaching of mathematics. As mathematics teachers trainers, we show in this work our proposal of teacher training model in which we develop the mathematical sense through: the identification of capabilities in solving school mathematical tasks, as well as in the identification of specific learning objectives for these tasks. In this model, we review the mathematical capabilities by relating them to different mathematical senses- number, spatial, measurement and stochastic-and exemplify tasks that allow their development in future primary school teachers. Likewise, these tasks develop some capabilities that future teachers could use in their profession, due to the model goes beyond the memorization of the senses and their capabilities, and aims to highlight a functional knowledge of mathematics.
\end{abstract}

Keywords: Mathematics for primary school teachers, mathematical sense, tasks, capabilities, learning objectives

\title{
1. INTRODUCCIÓN
}

Entendemos el Sentido matemático como el conjunto de capacidades relacionadas con el dominio en contexto de contenidos numéricos, geométricos, métricos y estadísticos, que permiten emplear estos contenidos de una manera funcional. Esta noción engloba cuatro sentidos correspondientes a cada bloque de contenido, denominados Sentido numérico (Sowder, 1992); Sentido espacial (Clements y Battista, 1992; Flores, Ramírez-Uclés y Del Río, 2015), Sentido de la medida (Shaw y Cliatt, 1989, Moreno, Gil y Montoro, 2015); y Sentido estocástico (Ruiz-Hidalgo y Serrano, 2015), basado en la idea de sentido estadístico (Watson, 2006; Batanero, Díaz, Contreras y Roa, 2013).

Esta idea hace que consideremos que la formación de profesores de matemáticas tiene que contribuir a que los futuros profesores aprecien qué significa enseñar matemáticas con sentido (matemático), y se preparen para lograr que su potencial alumnado aprenda matemáticas con sentido. Entendemos que 
"aprender matemáticas con sentido consiste en atender a sus usos en contexto y ofertar propuestas a las cuestiones que de ello se deriven" (Rico, Flores y Ruiz-Hidalgo, 2015, p.51). El origen de esta consideración arranca de apreciar que las matemáticas son una ciencia cultural, que permite pensar, entender y actuar en los problemas del entorno que tienen que ver con la cantidad, la forma, el tamaño y la incertidumbre aleatoria (OECD, 2016). Es por ello que se plantea una enseñanza funcional de las matemáticas, que haga predominar y dar sentido a los conceptos en resolución de problemas o tareas en contexto, frente al aprendizaje de destrezas o algoritmos en situaciones descontextualizadas (Rico y Díez, 2011). Establecemos así que es necesario disponer y desarrollar el sentido matemático para llegar a ser matemáticamente competente (Rico, Flores y Ruiz-Hidalgo, 2015).

Ante esta situación, en diversos cursos de formación de profesores de matemáticas, nos venimos planteando la necesidad de convertir la idea de sentido matemático en un foco de atención prioritario (Flores y Rico, 2015; Van de Walle, Karp y Bay-Williams 2013), tanto por constituir un objetivo de la enseñanza que cursarán los futuros maestros, como por originar un conocimiento didáctico del contenido matemático, que no se limite a aportar un conocimiento memorístico de resultados de investigaciones educativas sobre la enseñanza de las matemáticas. Este tipo de conocimiento está enfocado en la toma de decisiones en los procesos de enseñanza (Herbst, 2018) y en los procesos de reflexión en el contexto de formación de profesores (Piñeiro y Flores, 2018).

La forma con la que se abordan los cursos de formación matemática del Grado de Educación Primaria se centra en suscitar que los futuros profesores se cuestionen sobre cómo enseñar matemáticas con sentido. Este propósito se alcanza en tres etapas, que ayudan a definir las finalidades de los cursos que se incluyen en el plan de estudios:

1) a través de profundizar en el conocimiento del significado de los conceptos matemáticos escolares, que se puede concretar en identificar y organizar situaciones, contextos, fenómenos y modos de uso de cada concepto (Ruiz-Hidalgo, 2016);

2) mediante el desarrollo del conocimiento matemático con sentido de los estudiantes para profesor; y

3) como provisión de herramientas para llevar a cabo una enseñanza significativa en su vida profesional futura. 
En este trabajo nos centramos en la segunda etapa y mostramos algunas experiencias que estamos llevando a cabo para lograr dos propósitos relacionados con ella:

1) la identificación de capacidades en la resolución de tareas matemáticas escolares, y

2) la formulación y estudio de objetivos de aprendizaje específicos para que dichas tareas afronten un aprendizaje matemático con sentido.

Este trabajo se estructura alrededor de estos dos propósitos. En primer lugar mostramos un ejemplo de cómo favorecer que el alumnado del grado en Educación Primaria (el futuro docente) desarrolle sentido matemático de medida. Posteriormente mostramos cómo contribuimos a que el futuro profesor comprenda la noción de sentido estocástico, apreciando las necesidades informativas y de acciones, asociadas a las capacidades matemáticas (o componentes) que describen este sentido, para resolver un problema concreto. Por último, presentamos una experiencia para favorecer que el futuro profesor diseñe objetivos de aprendizaje, tendentes a lograr que sus alumnos desarrollen sentido numérico.

\section{EL PLAN DE FORMACIÓN EN MATEMÁTICAS EN EL GRADO DE EDUCACIÓN PRIMARIA}

El proceso de formación inicial de profesores de matemáticas tiene que abordar tanto las condiciones actuales de los candidatos a profesor, como las expectativas profesionales que tendrán que abordar en su desempeño futuro. A tal fin, en la Facultad de Ciencias de la Educación de la Universidad de Granada (España) se plantea una formación inicial de maestros en el área de matemáticas que lleve a los estudiantes a profundizar en el contenido matemático de primaria, arrancando de una visión funcional de la educación matemática, lo que conduce a suministrar herramientas para profundizar en el conocimiento, con intención práctica. Los estudiantes del grado deben llevar a cabo análisis didáctico de los contenidos matemáticos.

Por análisis didáctico de un contenido matemático escolar entendemos un método para escudriñar, estructurar e interpretar, dentro de un marco curricular, los 
contenidos didácticos de las matemáticas escolares, con el propósito de su planificación, su implementación en el aula y su evaluación (Rico, 2016a).

Consideramos el método conocido como análisis didáctico (Rico, Lupiáñez y Molina, 2013) en su faceta de herramienta para la elaboración de unidades didácticas de matemáticas (Ruiz-Hidalgo y Fernández-Plaza, 2013), estructurado en cuatro tipos de análisis:

- Análisis de contenido, centrado en describir y establecer los diferentes significados que tienen las nociones matemáticas.

- Análisis cognitivo, donde se estudian aspectos del aprendizaje de las matemáticas por parte de los escolares.

- Análisis de instrucción, enfocado en las cuestiones relacionadas con la instrucción, su planificación e implementación.

- Análisis de evaluación, cuyo fin es valorar el proceso de aprendizaje y los logros alcanzados.

El currículo de la formación inicial del maestro se relaciona con la idea de que el conocimiento didáctico tiene el papel de herramienta funcional, necesitando revisar investigaciones sobre la enseñanza y aprendizaje del contenido, para tener en cuenta apreciaciones que repercutan en su aprendizaje, como para determinar las dificultades de aprendizaje más habituales (Flores, 2018).

Realizar el análisis del contenido matemático (Rico, 2016b), lleva a comprender el significado de dicho contenido, mediante una profundización que aprecie y organice sus estructuras conceptuales, los sentidos, situaciones, usos y problemas que ayudan a resolver el contenido, así como la forma en que se representan (sistemas de representación y modelos). Fruto de este análisis de los contenidos de matemáticas de primaria, surge la materia que se denomina "Bases Matemáticas para la Educación Primaria", que se imparte en primer curso del grado de Maestro de Educación Primaria y que se concreta en un libro titulado Matemáticas para maestros de Educación Primaria (Segovia y Rico, 2011).

Una vez profundizado en el significado del contenido matemático, se pueden pasar a examinar los aspectos relativos a la enseñanza de dicho contenido, las expectativas y limitaciones de aprendizaje previsibles en los estudiantes de primaria, realizando un análisis cognitivo del contenido. Es en este momento en que se aborda la dimensión funcional del currículo de matemáticas de primaria (Rico y Díez, 2011), que lleva a apreciar la importancia de dar sentido al 
aprendizaje de las matemáticas. Todo esto dentro de un curso destinado a realizar el análisis cognitivo de los contenidos matemáticos, desarrollado en un libro de referencia titulado "Enseñanza y aprendizaje de las matemáticas en Educación Primaria" (Flores y Rico, 2015).

El concepto de sentido matemático aparece como un punto crucial del estudio cognitivo de los contenidos matemáticos (Lupiáñez y Rico, 2015; Rico, 2015). Durante diversos cursos en que hemos planteado el proceso, apreciamos la importancia de cubrir dos etapas para contribuir a que el estudiante valore en su justa medida el sentido matemático: mejorar su propio sentido matemático y apreciar en qué consiste y cómo se contempla en las tareas matemáticas escolares habituales en la educación primaria.

Para mostrar cómo abordamos estas dos etapas, comenzamos por describir el proceso formativo que pretende ayudarles a desarrollar el sentido matemático, describiendo un ejemplo basado en el sentido de la medida. A continuación, presentamos ejemplos de tareas para que los futuros profesores identifiquen los componentes del sentido de la medida, sentido estocástico y definan y organicen objetivos de aprendizaje para desarrollar el sentido numérico.

Todos los ejemplos se han desarrollado en sesiones de trabajo práctico de la asignatura "Enseñanza y aprendizaje de las matemáticas en Educación Primaria", en las que los futuros profesores trabajan en grupos de cuatro miembros de manera supervisada dentro del aula. Las sesiones prácticas incluyen el trabajo autónomo fuera del aula para completar la tarea solicitada y redactarla. Estas sesiones complementan a las lecciones teóricas en las que se describen, discuten y ejemplifican los componentes de cada uno de los sentidos. El curso total está compuesto por 15 sesiones teóricas (dictadas a grandes grupos de unos 60 futuros profesores) y 15 prácticas (dictadas a grupos de 20 futuros profesores), de las cuales 5 teóricas y 10 prácticas se dedican al estudio del sentido matemático. En las sesiones prácticas, los 20 futuros maestros se organizan en pequeños grupos (4 miembros) que realizan tareas como las que ejemplificamos a continuación. Tras trabajar todos los sentidos (numérico, espacial, medida, estocástico), se les propone un trabajo final que se describe en el punto 5.

\section{DESARROLLAR EL SENTIDO DE LA MEDIDA}

La idea de sentido de la medida que se trabaja en el curso aparece en el capítulo correspondiente del libro de texto (Moreno, Gil y Montoro, 2015), y enfatiza tres 
componentes o capacidades específicas y etapas para aprender con sentido la medida: captar la magnitud, llevar a cabo medidas y desarrollar estrategias y actividades de estimación. En diversos cursos hemos empleado una tarea práctica para mejorar el desarrollo del sentido de la medida (Fernández, 2011) de los futuros profesores, al determinar con sentido la dosis de un tratamiento. En el cuadro 1 aparece el enunciado de esta tarea.

Cuadro 1. Tarea "Fluidasa"

Resuelve con sentido la situación siguiente:

"FLUIDASA SOLUCIÓN" es un jarabe, cuyo principio básico es la "mepifilina", que está indicado para la tos en pacientes con bronquitis. En el prospecto, respecto a las instrucciones de administración, indica: 1 medida de $5 \mathrm{ml}$ contiene aproximadamente $25 \mathrm{mg}$ de "mepifilina". La dosis normal es de $8 \mathrm{mg}$ por $\mathrm{kg}$ de peso y día, fraccionada en 3 tomas. Rocío es una niña de 6 años que pesa aproximadamente 19 kg, ¿cuántas cucharaditas de las de café (5 $\mathrm{ml}$ ) hay que darle en cada toma?

Durante una sesión práctica de trabajo los profesores en formación, en grupos de 4, abordan la resolución de esta tarea. Usualmente muestran disposición a tomar los datos, buscar las operaciones y resolverlas, llegando a soluciones (correctas o no), que generalmente emplean números decimales de cucharadas en cada toma.

La puesta en común de las soluciones suele confrontar las respuestas fraccionarias con las que abordan la cantidad de cucharadas de una manera menos precisa. Es en este momento en que proponemos que examinen las magnitudes y los objetos que están presentes en el problema. Con cierta facilidad aparecen tres magnitudes (capacidad de cuchara, masa del niño y duración, edad del niño). Las otras dos magnitudes, masa de mepifilina y volumen de la disolución, suelen ser más complicadas de apreciar, al venir dadas por tasas que relacionan la masa de mepifilina con el volumen de disolución y la masa de mepifilina con el peso del paciente, pero son fundamentales para comprender el problema en toda su extensión.

En esta fase se aborda el componente conceptual del sentido de medida, concretamente centrada en identificar cuál es la cualidad que se aprecia de cada objeto, distinguiendo las magnitudes que son pertinentes (dejando de lado la edad, aun reconociendo que es pertinente habitualmente en la determinación de una dosis, pero que en este caso no disponemos de información al respecto, 
y que esta relación no suele ser de proporcionalidad, tal como la que relaciona el peso de la niña con la dosis, sino una relación escalonada).

Apreciar las unidades de medida de cada magnitud (estableciendo o no correspondencias entre masa y peso, según proceda), separando las de capacidad y volumen, pero captando la relación entre ambas, es el paso siguiente. De este estudio se llega a clarificar la medida aproximada, y a reconocer el empleo cotidiano de una unidad de medida no estándar (la cucharada), apreciando la escasa precisión que lleva a hacer estas apreciaciones. Una actividad de medida de diversas cucharadas podría hacer más completa esta parte de la resolución, comenzando por ordenar de manera intuitiva la capacidad de diversas cucharas, comprobando dicha ordenación, identificando la influencia de la tensión superficial y cómo varía la capacidad según se colme o no la cuchara, y según el líquido empleado. Este proceso de estimación y medida puede colaborar a desarrollar el tercer componente del sentido de la medida, es decir, la estimación, tanto para apreciar la diversidad de medidas de capacidad, como comenzar a interiorizar su capacidad, y percibiendo el interés en estimar sobre el medir en situaciones como la presentada.

Después de estas fases se puede trabajar con la resolución "con sentido matemático" de esta tarea, apreciando que la precisión permite reducir la dosis a dos cucharadas en cada toma, sin por ello faltar a las apreciaciones que figuran en el prospecto. Durante el proceso se han enfatizado los componentes que caracterizan el sentido de medida, especialmente para apreciar la importancia de tomar en consideración todas las facetas implicadas. La siguiente sesión de trabajo se dedica a apreciar los componentes en diversas situaciones, tanto para afrontarlas y resolverlas, como para explicarlos.

\section{ELABORACIÓN DEL LISTADO DE LOS COMPONENTES DE CADA SENTIDO}

Para evitar la memorización innecesaria de los componentes de cada sentido y centrar el esfuerzo en desarrollar lo que estos significan y cuáles son los indicadores de las actividades que los caracterizan, se les pide que elaboren un esquema exclusivamente con la denominación de los componentes, a partir de referencias aportadas en clase. El esquema debe incluir "indicadores" que le permitan analizar las tareas y discriminar si se pone en juego cada componente. 
Se muestra en el anexo I un ejemplo de esquema elaborado por un estudiante, con el listado de los componentes de los cuatro sentidos.

\subsection{LOS FUTUROS PROFESORES IDENTIFICAN LOS COMPONENTES DEL SENTIDO ESTOCÁSTICO}

Durante la sesión de trabajo práctico destinada a que los futuros profesores aprecien el concepto de sentido estocástico (Ruiz-Hidalgo y Serrano, 2015), hemos empleado la tarea matemática escolar "Autobús o caminar", que aparece en el cuadro 2, contextualizada en Granada y su sistema de transporte urbano.

Cuadro 2. Tarea "Autobús o caminar"

Examina tu sentido estocástico contestando razonadamente la siguiente cuestión: Cuando se viene a la Facultad de Ciencias de la Educación desde la Plaza Albert Einstein, ¿̇es mejor siempre tomar la línea U3 de autobuses que venir caminando?

Informaciones: La línea U3 de autobuses de Granada tiene 3 paradas intermedias entre el punto de salida (Plaza Einstein) y la Facultad de Ciencias de la Educación. Cada parada coincide con un semáforo y el tiempo de espera en cada parada es el mismo que dura el semáforo. Los horarios de salida de Plaza Einstein están establecidos por la empresa concesionaria, y hay que buscarlos en la información correspondiente.

Resolución (en el seminario): En clase deberás estudiar la situación, examinando:

A: Qué parte del estudio corresponde a un proceso aleatorio y cuál a un determinista

B: Cómo puedes cuantificar la probabilidad en los apartados aleatorios

C: Qué datos necesitas buscar y cómo obtenerlos

D: Cómo puedes resumir los datos para extraer informaciones útiles

E: Cómo llevar a cabo el estudio completo.

Se han previsto una serie de informaciones que te van a ayudar a realizar el estudio, cada una de ellas corresponde a uno de los apartados anteriores. Conforme se te van indicando se te ayuda a desarrollar el componente correspondiente del sentido estocástico.

Trabajo del grupo a entregar:

a) Resolución: Realizar

a1) Un cuadro que exprese los tiempos medios de duración del trayecto, según la hora.

a2) Un informe que responda razonadamente a la pregunta (Cuando se viene desde la Plaza de Einstein, ¿̇es mejor siempre tomar la línea U3 de autobuses que venir caminando?), y que aclare las condiciones y decisiones adoptadas para llegar a la solución.

b) Demuestra que comprendes la idea de sentido estocástico

Identifica justificadamente todos los componentes del sentido estocástico que se ponen de manifiesto al realizar la tarea, señalando cómo se aplica cada uno. 
Para llevar a cabo esta tarea, se ha previsto que los futuros profesores aprecien los datos que tienen y los que les faltan. Cuando empiezan a detectar necesidades se les suministra una baraja de informaciones, compuesta por cuatro colecciones, cada una correspondiente a una de las letras indicadas (A: Qué parte del estudio corresponde a un proceso aleatorio y cuál a un determinista; B: Cómo puedes cuantificar la probabilidad en los apartados aleatorios; C: Qué datos necesitas buscar y cómo obtenerlos; D: Cómo puedes resumir los datos para extraer informaciones útiles; y E: Cómo llevar a cabo el estudio completo). Se entrega una colección a cada integrante del equipo, quienes deben mirar las informaciones que les ayuden a resolver el problema. Con ello se pretende que se produzca una interpretación cruzada de informaciones, en la que vayan completándolas y teniendo presente el origen (el componente) de cada información y la dependencia entre ellas. En el anexo II figura la baraja de informaciones suministradas.

El siguiente paso, como se ha descrito en el ejemplo anterior y que se repite en todas las actividades sobre sentidos, consiste en identificar componentes de cada sentido que contribuyen a desarrollar en tareas matemáticas escolares de libros de texto y en diseñar otras actividades dirigidas al alumnado de Educación Primaria que contribuyan al mismo fin.

El proceso de compartir diversas clases de información, relacionándolas entre sí, hasta construir un mapa de relaciones, lleva a darle presencia al sentido matemático como finalidad de enseñanza. El problema siguiente consiste en definir objetivos de aprendizaje que tengan en cuenta tanto el significado de los contenidos matemáticos, como el desarrollo del sentido matemático en el alumnado de primaria. Esto lo abordamos en el apartado 6, tomando casos particulares relacionados con el sentido numérico.

\section{DISEÑO Y COEVALUACIÓN DE ACTIVIDADES PARA DESARROLLAR SENTIDO MATEMÁTICO}

Una vez realizada la aproximación inicial, desarrollo, organización e identificación de los componentes que describen cada sentido matemático, presentada en este artículo mediante las actividades ejemplificadas, se propone a los futuros maestros como trabajo final práctico la selección de uno o varios componentes y el diseño justificado de una propuesta de actividad para el alumnado de Educación Primaria que contribuya a desarrollar dichos componentes. 
Puesto que nuestros estudiantes no pueden llevar a la práctica del aula de primaria cada propuesta, el trabajo de cada grupo se desarrolla y evalúa en dos fases. En la primera, la actividad planteada por cada grupo es revisada por otro grupo de trabajo que realiza un informe "borrador" durante la hora de clase, en el que se evalúa la actividad atendiendo a los aspectos de forma $y$, específicamente, al grado en que se cubren las capacidades que plantean los compañeros, analizando críticamente la justificación que proporcionan. Completan el análisis de la tarea reflejando otros componentes destacados y planteando mejoras. En la segunda fase cada grupo, una vez recibido el informe, elabora una nueva versión del trabajo en el que modifica su actividad y el análisis de los componentes. Esta producción es empleada en la evaluación del grupo por el profesor de la materia, quien también valora el informe elaborado sobre el trabajo de otro grupo.

Como ejemplo, en relación con el sentido de la medida, en el cuadro 3 se muestra una actividad planteada por un grupo de futuros maestros. El cuadro 4 incluye la crítica realizada por otro equipo de trabajo.

Cuadro 3. Actividad y capacidades indicadas por un grupo

Completa la siguiente tabla midiendo con un bolígrafo BIC la pizarra, el pupitre (largo y ancho), una libreta, la pata de una silla y la puerta del aula. A continuación, haz la estimación del ancho y largo del aula mediante pasos

\begin{tabular}{|l|l|l|}
\hline Mide: & Mide: & Mide: \\
\hline Mide: & Mide: & Mide: \\
\hline
\end{tabular}

Capacidades que se trabajan (respuesta dada por uno de los grupos):

Ejecución del proceso de medir. Seleccionar la unidad adecuada, estándar o no estándar, procedimiento e instrumentos adecuados. Usar fórmulas cuando sea apropiado. Expresar adecuadamente el resultado. 
Cuadro 4. Crítica realizada por otro grupo de futuros profesores a la tarea del cuadro 3.

La actividad presenta las siguientes fortalezas:

- Desarrolla la capacidad que se menciona.

- Las unidades no convencionales son ideales para realizar medidas.

Sin embargo, presenta las siguientes debilidades:

- No se justifica cómo trabaja dicha actividad esa capacidad.

- También falta añadir que dicha actividad contribuye al desarrollo de la capacidad de estimación.

- No se contrasta la medición con el empleo de instrumentos estandarizados para comparar las medidas realizadas con unidades no convencionales. Por ejemplo, si el aula mide de largo 17 pasos de Juan y 21 pasos de Luis, no hay referente estándar respecto del cual averiguar qué medición ha sido más precisa.

Como sugerencia de mejora, se propone incluir cuestiones destinadas a mostrar que se obtienen diferentes medidas cuando estamos trabajando con la misma unidad de medida no estándar (las partes del cuerpo de cada alumno tienen diferente tamaño) y por tanto se hace necesario el uso de unidades estándar.

Finalmente, esta actividad se puede trabajar en parejas para fomentar la motivación y la colaboración.

La tarea propuesta en el cuadro 3 se divide en dos: la primera es de medida directa en la que la unidad de medida está determinada. La segunda tarea, por su parte, es de estimación aunque no requiere tomar decisiones sobre medir o aproximar. Así, las capacidades a desarrollar en ambas son, fundamentalmente "Aplicar el procedimiento e instrumento adecuados para efectuar dicha medición ya sea exacta o aproximada" y "Expresar adecuadamente el resultado de la medición" (ver anexo I).

En la capacidades indicadas por los futuros profesores (cuadro 3, transcrito literalmente), se perciben algunas deficiencias como el énfasis en capacidades secundarias: "Seleccionar la unidad adecuada, estándar o no estándar" (que está determinada por el enunciado) y "Usar fórmulas cuando sea apropiado" (que se refiere al uso de fórmulas para medidas indirectas). Asimismo, aparecen carencias relacionadas con el contenido matemático expresado por los futuros profesores como la ausencia de dimensiones o magnitudes (longitud/superficie/ volumen) que han de medirse en algunos de los objetos señalados.

Por su parte, la crítica del cuadro 4, acierta parcialmente en la valoración pues menciona que se desarrollan las capacidades mencionadas, pero no indica si todas o sólo algunas. La afirmación sobre el uso de unidades no 
convencionales como ideales no es apropiada. Sin embargo, aciertan en proponer la capacidad de estimación como implicada en la tarea, y dedicar esfuerzo a realizar aclaraciones sobre el proceso de medida mediante la estimación y la diferencia entre unidades estándar y no estándar, lo que manifiesta que han identificado estos procesos como fundamentales de la tarea, y sugieren a sus compañeros que deberían describirlos con más detalle.

\section{DEFINIR OBJETIVOS DE APRENDIZAJE}

Dentro de la realización del análisis cognitivo de un contenido, los futuros maestros tienen que definir expectativas de aprendizaje, en forma de objetivos específicos. Estos objetivos deben recoger la idea de sentido matemático. ¿Cuáles y cómo deben ser los objetivos de aprendizaje para que los alumnos aprendan matemáticas con sentido?

Los sentidos matemáticos nos hacen prestar atención a todos los componentes necesarios para el aprendizaje de un concepto, concediendo igual atención a la comprensión que al aprendizaje de destrezas y técnicas matemáticas. Es decir, el sentido matemático requiere:

a) Equilibrio entre conocimiento conceptual y procedimental.

b) Cubrir el máximo de componentes o respetar la importancia de cada uno de ellos.

\subsection{OBJETIVOS CONCEPTUALES Y PROCEDIMENTALES:}

Es relativamente fácil establecer objetivos cuando tienen intención procedimental, ya que es sencillo identificar qué queremos que el alumno aprenda, en qué orden, con qué pasos, etc. Por ejemplo, si queremos que aprenda el algoritmo tradicional de la resta, no es difícil encontrar etapas:

- Restar números de una cifra (5 - 2; 8 - 6, etc.).

- Restar de un número de dos cifras otro de una cifra, primero sin llevarse (15 - 3; $27-6 ; 28-8)$, luego llevándose (13 - 9; 26 - 7, etc.), terminando con las restas a múltiplos de 10 (20 - 7, $30-9$, etc.), colocando siempre 
los números en papel cuadriculado, para alinear las unidades del orden correspondiente.

- Restar números de dos cifras, primero sin llevada (36-24; 43 - 31), luego llevándose (36 - 27), para terminar con múltiplos de 10 (40 - 24; 35 - 20), colocando los números en papel cuadriculado.

- Aumentar el número de cifras de los números, incluyendo aquellos que no tienen igual cantidad de cifras $(123$ - 85).

Esta consideración permite formular los siguientes objetivos del aprendizaje del procedimiento:

1) Obtener el resultado de una resta de números de una cifra:

a) Recordando las sumas correspondientes

b) Determinando cuánto hay que sumar al minuendo para llegar al sustraendo

2) Obtener el resultado de una resta de números de dos cifras menos números de una cifra:

a) Sin llevada

b) Con llevada

No es tan fácil definir objetivos correspondientes a capacidades de comprensión de conceptos: el concepto de resta, por ejemplo. ¿Qué es comprender?, ¿cómo podemos expresar los objetivos conceptuales para evitar que se conviertan en objetivos de memorización?

Según la Real Academia de la Lengua (RAE), comprender es entender, alcanzar. En Psicología, el concepto de comprensión es la acción de percibir el significado de algo, se identifica con una actividad cognoscitiva que comparte elementos con el conocimiento racional y con sus técnicas interpretativas, su interés es interpretar el sentido de las cosas (Hiebert y Carpenter, 1992).

De aquí que interpretemos que un alumno comprende la resta cuando:

- Resuelve los diversos problemas que corresponden a la resta (todos los tipos de problemas determinados en el análisis de contenido, cuando estudiamos su significado: cambio, combinación, comparación; en todas las situaciones -incógnita en la transformación y en la cantidad inicial, incógnita en uno de los sumandos, incógnita en el elemento de comparación aditiva, o en alguno de los referentes, en la comparación sustractiva). 
- Pasa de una forma de representación a otra (al plantearle una resta de manera vertical, la identifica con otra horizontal, 12 - 9, piensa en una situación con significado que corresponda a esta resta - tengo 12 cromos y pierdo 9, cuántos me quedan -, puede expresarlo por medio de otras representaciones, desde los objetos, el ábaco, etc.).

- Es capaz de interpretar la situación de diversas formas, buscando la más sencilla cuando tiene dudas al respecto (12 - 9 también puede interpretarse como averiguar cuántos pisos tengo que subir, si estoy en el 9 y quiero ir al 12, que es lo mismo que averiguar cuántos tengo que subir si estoy en el 10 y quiero ir al 13, con lo que aprecio más fácilmente que es 3; por tanto en la aplicación del procedimiento de resta con llevada tiene que salir 3; u otras estimando: 12 está en la segunda decena, tendré que subir menos de 10 pisos, estimando, el tamaño del resultado).

Los análisis realizados hasta el momento deben facilitar al futuro profesor saber qué quiere lograr en su enseñanza, y definir objetivos que vayan en la dirección prevista, es decir, que estén encaminados a que el alumno aprenda matemáticas con sentido.

En la formación de profesores tenemos que enseñar a definir objetivos y hacerles apreciar en qué grado los objetivos planteados recogen lo más importante de los sentidos, así como los elementos que contribuyen a ello, es decir, a comprender, además de a aplicar técnicas.

\subsection{ANÁLISIS DE LOS OBJETIVOS PROPUESTOS POR LOS FUTUROS PROFESORES}

Para disponer de criterios con los que revisar los objetivos formulados por futuros profesores, presentamos un proceso que les permite examinar en qué grado sus objetivos plantean el logro de un aprendizaje con sentido de las matemáticas, respondiendo a cuestiones como las siguientes:

- Qué aspectos del contenido estamos enfatizando, ¿sólo los procedimentales? ¿también los conceptuales?, ¿¿n qué proporción?, ¿̇son adecuados para alcanzar el sentido matemático correspondiente?

- Qué componentes del sentido matemático se están teniendo en cuenta ¿están todos?, ¿aparece alguno más que los demás?, ¿somos conscientes y es lo que interesa? 
- Qué aspectos de significado estamos teniendo en cuenta ¿se recogen los adecuados para que el alumno llegue a comprender?, ¿es conveniente añadir alguno?

- Qué elementos de las representaciones se han atendido, ¿̇son suficientes para que el alumno interprete unos en función de los otros?, ¿favorecen que pase de la representación simbólica a representaciones materiales?, ¿he previsto que se utilicen representaciones gráficas que permitan pasar de simbólicas a materiales en ambos sentidos?

Para responder a estas cuestiones, es decir, para poder juzgar si los objetivos definidos son adecuados, si están definidos de manera funcional, empleamos la tarea del cuadro 5 en esta parte final de los cursos de formación de profesores. En ella revisan los objetivos formulados y los rectifican a partir de apreciar en qué proporción aparece cada componente y en qué grado coincide con sus intenciones formativas según ciclo.

Cuadro 5. Los colores. Tarea

Consideramos esta asignación de los colores del parchís:

Concepto: Rojo

Procedimientos: Azul

Representaciones: Amarillo

Usos/Fenómenos: Verde

1. Utiliza estos colores para el mapa conceptual del análisis de contenido

2. Asigna un color a cada uno de los objetivos, según esté relacionado con alguno de los elementos del análisis de contenido. Si está asociado a varios, únicamente utiliza el que esté más relacionado (para ello, piensa en qué tiene que realizar el alumno para que estés seguro que lo has logrado)

3. Asigna a cada objetivo, los componentes de los sentidos que estén asociadas a él (pueden ser varias). Utiliza esta codificación:

- Sentido Numérico (SN1=reconocer cuándo y cómo usar los números, SN2: componente 2....)

- Sentido Espacial (SP1: elementos geométricos, SP2: componente 2....)

- Sentido de la medida (SM1: reconocimiento de cualidades medibles, SM2: componente 2, etc.)

- Sentido estocástico (SE1: reconocer situaciones aleatorias, SE2: componente 2, etc.)

Observa el siguiente ejemplo, donde se ha marcado como procedimental este objetivo y se ha indicado la componente SP2.

Ejemplo. Objetivo: Construir un cuadrado a partir de uno de sus lados (SP2: relaciones geométricas, visualización)

4. Reflexiona sobre las siguientes cuestiones:

¿Se reflejan todos los colores en tus objetivos? ¿Qué color prevalece? ¿Por qué?

¿Se reflejan todos los contenidos en tus objetivos? ¿Crees que has "barrido" con tus objetivos el análisis de contenido? ¿Has utilizado todos los componentes de los sentidos relacionados? ¿Cuáles faltan? Añade un objetivo o reformula los que tienes para considerarlas. 


\section{REFLEXIONES FINALES}

Hemos presentado algunas tareas utilizadas en la formación de maestros de educación primaria para desarrollar la noción de sentido matemático. Esta noción queda concretada en los sentidos numérico, espacial, de la medida y estocástico.

Con la tarea Fluidasa (cuadro 1) hemos ejemplificado momentos y situaciones en las que se pueden poner en juego los conjuntos de capacidades relacionadas con los componentes del sentido de la medida: entender la noción de magnitud, desarrollar los procedimientos de medida y desarrollar estrategias de estimación.

La tarea Autobús o caminar (cuadro 2) nos ha servido para ejemplificar el trabajo del sentido estocástico, concretamente para que el estudiante identifique las capacidades relacionadas con los componentes tratamiento de datos (captura, organización) y con la toma de decisiones a partir de los datos.

Como se ha mostrado en los cuadros 3 y 4 el diseño de las tareas durante la formación de profesores incentiva la comunicación y retroalimentación entre diferentes grupos de trabajo, promoviendo su análisis crítico respecto de los componentes del sentido de la medida.

Además de resolver tareas para desarrollar el sentido matemático y comprender sus componentes, también contribuye a desarrollar el sentido matemático al proponer y analizar objetivos de aprendizaje de una tarea. Así, la tarea Los cuatro colores (cuadro 5) permite a los futuros maestros reflexionar sobre las metas de una tarea. Proponer objetivos de aprendizaje, lejos de ser una labor fácil, pasa por la identificación, distinción y selección de capacidades conceptuales y procedimentales, que hemos apreciado en nuestra experiencia como formadores, y resulta una actividad compleja para los futuros profesores.

Las tareas presentadas persiguen una comprensión del significado del sentido matemático que va más allá de la memorización de sus componentes, resaltando un conocimiento funcional que permita a los futuros profesores identificarlos y proponer tareas que las desarrollen. Para destacar el interés que tenemos en que usen de manera práctica los componentes, sobre su memorización, se permite a los estudiantes que diseñen su propio resumen con la denominación de los componentes y que dispongan de él en todo momento, incluidas las tareas de evaluación.

En relación con los referentes teóricos, en este trabajo hemos analizado el potencial de las tareas propuestas para que los estudiantes comprendan 
el concepto de sentido matemático. Consideramos que una continuación en futuros trabajos sería tratar de analizar las producciones de los estudiantes y estudiar la evolución en su comprensión de los componentes.

\section{AGRADECIMIENTOS}

Este trabajo fue respaldado por el Ministerio de Ciencia y Tecnología de España en el marco de los Proyectos Nacionales I+D EDU2015-70565-P y EDU201675771-P, y el Grupo FQM-193 del III Plan Andaluz de Investigación (PAIDI).

\section{REFERENCIAS}

Batanero, C., Díaz, C., Contreras, J. M. y Roa, R. (2013). El sentido estadístico y su desarrollo. Números, 83, pp. 7-18.

Clements, D. H. y Battista, M. T. (1992). Geometry and spatial reasoning. In D. A. Grouws (Ed.), Handbook of research on mathematics teaching and learning: A project of the National Council of Teachers of Mathematics (pp. 420-464). New York, NY, England: Macmillan Publishing Co, Inc.

Fernández, F. (2011). El sentido de la Medida. Lección inaugural del curso 2011-2012 de la Facultad de Ciencias de la Educación. Granada: Universidad de Granada.

Flores, P., Ramírez-Uclés, R. y Del Río, A. (2015). Sentido espacial. En P. Flores y L. Rico (coords.), Enseñanza y aprendizaje de las matemáticas en Educación Primaria (pp. 127-146). Madrid: Pirámide.

Flores, P. y Rico, L. (2015). Enseñanza y aprendizaje de las matemáticas en Educación Primaria. Madrid: Pirámide.

Flores, P. (2018). ¿Por qué multiplicar en cruz? Formación inicial de profesores de primaria en el área de Matemáticas. Unión, 53, 9-29.

Herbst, P. (2018). Teoría y métodos para la investigación de la racionalidad de la práctica en la enseñanza de las matemáticas. Educación Matemática, 30(1), pp. 9-46.

Hiebert, J. y Carpenter, T. (1992). Learning and teaching with understanding. En D. Grouws (Ed.), Handbook of research on mathematics teaching and learning: A project of the National Council of Teachers of Mathematics (pp. 65-97). New York, NY, England: Macmillan Publishing $\mathrm{Co}$, Inc. 
Lupiáñez, J. L. y Rico, L. (2015). Aprender las matemáticas escolares. En P. Flores y L. Rico (Coords.), Enseñanza y aprendizaje de las matemáticas en Educación Primaria (pp. 41-60). Madrid: Pirámide.

Moreno, M. F., Gil, F. y Montoro, A. B. (2015). Sentido de medida. En P. Flores y L. Rico (Eds.), Enseñanza y aprendizaje de las matemáticas en Educación Primaria (pp. 147168). Madrid: Pirámide.

OECD (2016). PISA 2015 Assessment and Analytical Framework: Science, Reading, Mathematic and Financial Literacy. PISA, OECD Publishing, Paris.

Piñeiro, J.L. y Flores, P. (2018). Reflexión sobre un problema profesional en un contexto de formación de profesores. Educación Matemática 30(1), 237-251.

Rico, L. (2015). Matemáticas escolares y conocimiento didáctico. En P. Flores y L. Rico (Coords.), Enseñanza y aprendizaje de las matemáticas en Educación Primaria (pp. 21-40). Madrid: Pirámide.

Rico, L. (2016a). Matemáticas y análisis didáctico. En A. Moreno y L. Rico (Coords.), Elementos de Didáctica de la Matemática para el profesor de Secundaria (pp. 85-100). Madrid: Pirámide.

Rico, L. (2016b). Significado de los contenidos matemáticos. En A. Moreno y L. Rico (Coords.), Elementos de Didáctica de la Matemática para el profesor de Secundaria (pp. 153- 174). Madrid: Pirámide.

Rico, L. y Díez, A. (2011). Las matemáticas y el maestro de Primaria. En I. Segovia y L. Rico (Coords.), Matemáticas para maestros de Educación Primaria (pp. 23-45). Madrid: Pirámide.

Rico, L., Flores, P. y Ruiz-Hidalgo, J. F. (2015). Enseñanza de las matemáticas con sentido. Uno. Revista de Didáctica de las Matemáticas, 70, pp. 48-54.

Rico, L., Lupiáñez, J. L. y Molina, M. (2013). Análisis didáctico en Educación Matemática. Metodología de investigación, formación de profesores e innovación curricular. Comares: Granada, España.

Ruiz, J. F. y Serrano, L. (2015). Sentido estocástico. En P. Flores y L. Rico (coords.). Enseñanza y aprendizaje de las matemáticas en educación primaria (pp. 169-184). Madrid: Pirámide.

Ruiz-Hidalgo, J. F. (2016). Sentido y modos de uso de un concepto. En L. Rico y A. Moreno (coords.), Elementos de didáctica de la matemática para el profesor de secundaria (pp. 139-151). Madrid: Pirámide.

Ruiz-Hidalgo, J. F. y Fernández-Plaza, J. A. (2013). Planificación de unidades didácticas en enseñanza secundaria mediante el uso del Análisis Didáctico. En L. Rico, J. L. Lupiáñez y M. Molina (Eds.), Análisis Didáctico en Educación Matemática. 
Metodología de investigación, formación de profesores e innovación curricular (pp. 231-251). Granada: Comares.

Segovia, I. y Rico, L. (2011). Matemáticas para maestros de educación primaria. Madrid: Pirámide.

Shaw, J. M. y M. J. P. Cliatt. (1989). Developing Measurement Sense. En P. R. Trafton y A. P. Shulte (ed.), New Directions for Elementary School Mathematics. 1989 Yearbook (pp. 149-155) Reston, VA: National Council of Teachers of Mathematics.

Sowder, J. (1992). Estimation and number sense. In D. Grouws (Ed.), Handbook of research on mathematics teaching and learning: A project of the National Council of Teachers of Mathematics (pp. 371-389). New York, NY, England: Macmillan Publishing Co, Inc. Van de Walle, J. A., Karp, K. S. y Bay-Williams, J .M. (2013). Elementary and Middle School Mathematics. Teaching developmentally. Pearson Education.

Watson, J. M. (2006). Statistical literacy at school: growth and goals. Mahwah, NJ: Lawrence Erlbaum Associates.

Dirección postal: Departamento de Didáctica de la Matemática Facultad de Ciencias de la Educación Campus de Cartuja, $\mathrm{s} / \mathrm{n}$

18071 Universidad de Granada, Granada, España

Teléfono: $\quad+34958241993$ 


\section{Anexo I}

\section{Capacidades/Componentes que caracterizan el Sentido numérico}

1. Reconocer cómo y cuándo usar los números

2. Discernir en qué ocasiones se ha de dar un valor exacto y en cuales un valor aproximado.

3. Detectar patrones y usar relaciones numéricas

3.1. Componer y descomponer números

4. Percibir la magnitud de los números

5. Realizar cálculos numéricos con procedimientos diferentes

5.1. Aplicar algoritmos diferentes al estándar

5.2. Desarrollar estrategias de cálculo mental

5.3. Realizar estimaciones razonables

5.4. Elegir el procedimiento más sencillo (debe haber dos procedimientos alternativos)

6. Conocer diferentes representaciones de los números y usar la más adecuada.

6.1. Emplear propiedades del sistema decimal de numeración

7. Resolver problemas con diversidad de estrategias (al menos dos)

8. Inventar problemas a partir de datos y operaciones propuestas

9. Detectar y corregir errores aritméticos

\section{Capacidades/Componentes que caracterizan el Sentido espacial}

1. Manejo de conceptos geométricos

1.1. Conocer e identificar propiedades de formas y figuras

1.2. Reconocer, aplicar y establecer relaciones geométricas (Clasificar atendiendo a diferentes criterios)

1.3. Movimientos: Aplicar movimientos a figuras y conocer sus propiedades.

2. Destrezas de visualización

2.1. Localización y Orientación

2.2. Visualización propiamente dicha 
Capacidades/Componentes que caracterizan el Sentido de la medida

1. Reconocer cualidades comparables y medibles de los objetos

2. Ejecutar el proceso de medir

2.1. Seleccionar la unidad de medida adecuada estándar o no estándar

2.2. Aplicar el procedimiento e instrumento adecuados para efectuar dicha medición ya sea exacta o aproximada

2.3. Usar fórmulas cuando sea apropiado

2.4. Expresar adecuadamente el resultado de la medición

3. Desarrollo de estrategias de estimación

3.1. Discernir situaciones en las que se requiere una medición exacta y otras en las que baste una estimación

Capacidades/Componentes que caracterizan el Sentido estocástico

1. Razonamiento probabilístico

1.1. Identificación de situaciones aleatorias

1.2. Cuantificación del grado de incertidumbre

2. Razonamiento estadístico

2.1. Búsqueda y obtención de datos

2.2. Resumen estadístico de la información

2.3. Realizar inferencias o sacar conclusiones coherentes 


\section{Anexo II. Baraja de informaciones suministradas para la tarea "Autobús o caminar"}

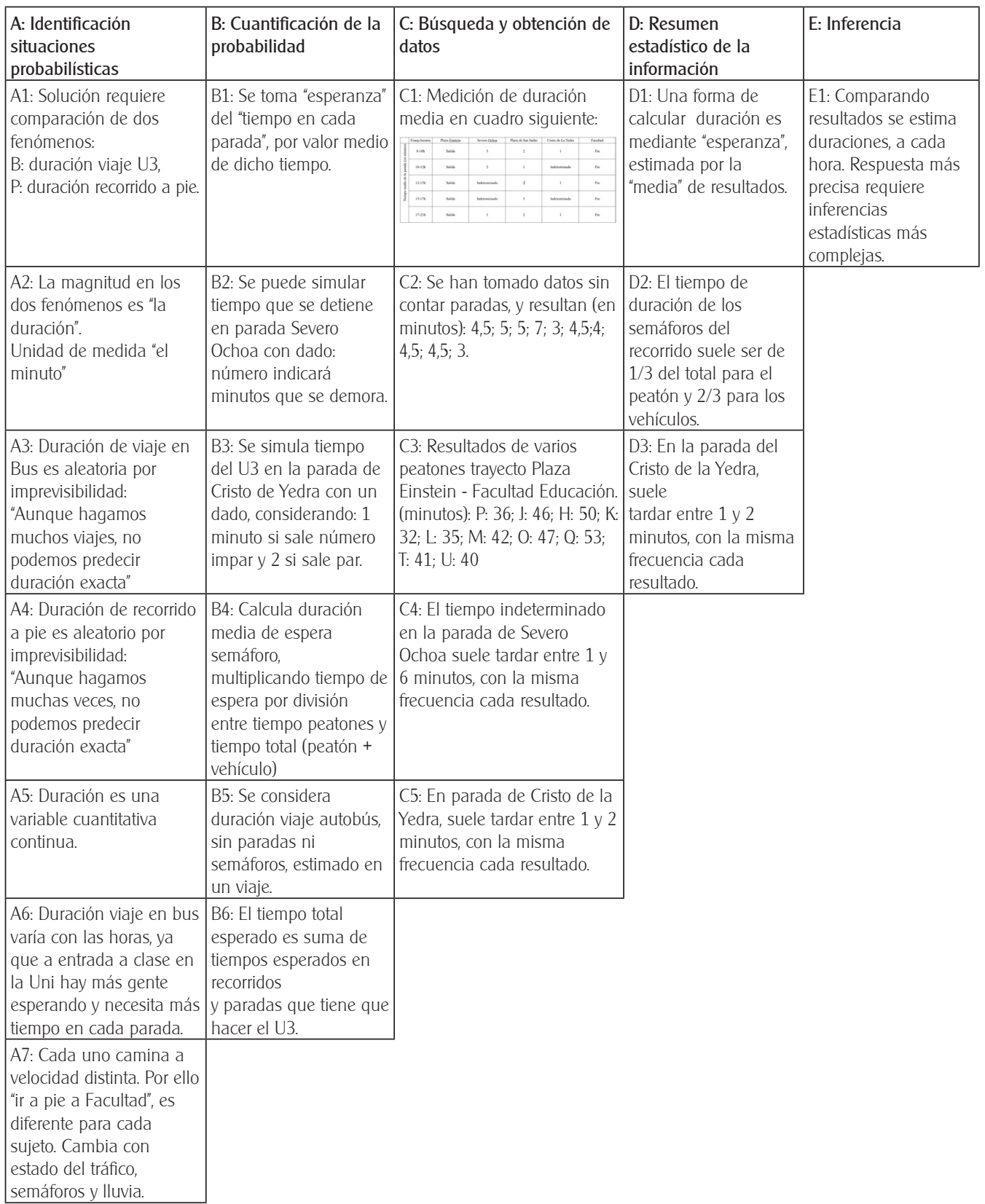

